\title{
Amplification of changes of a thin film's macromolecular structure into macroscopic reaction-diffusion patterns.
}

\author{
SUPPORTING INFORMATION
}

\author{
Agnieszka Bitner, Marcin Fiałkowski, Stoyan Smoukov, Christopher J. Campbell and \\ Bartosz A. Grzybowski
}

Northwestern University, Department of Chemical and Biological Engineering and Northwestern Institute for Complexity, 2145 Sheridan Rd, Evanston, Illinois 60208

\section{S1. Comparison with Networks Having One Type of Nodes.}

We have recently described a related system that used networks with one type of nodes to toggle between tile-centering (TC) and dual lattice (DL) reaction-diffusion patterns in response to the changes in the geometric parameters of the system (cf. Reference 8 in the text and Fig. S1 below). In that system, water gradients established in the network were such that: (i) there was less water near the gelatin surface than at the bases of the features; (ii) less near the borders of the edges than at their centers and, importantly, (iii) always less in the edges than in the nodes. The iron cations contained in the network could migrate into gelatin either "vertically" from the edges (to give a TC pattern) or first "horizontally" towards the nodes and only then into gelatin (to give a DL solution). The particular mode was determined by the relationship between characteristic times of lateral and vertical transport ( $\tau_{\text {esc }}$ and $\tau_{\text {closure }}$, respectively), and the TC/DL transition was governed by a dimensionless parameter $\eta=d / L$ (ratio of edge thickness to its length). The final $\mathrm{RD}$ pattern represented the TC transformation of the stamped network for $\eta<\eta^{*}$, and the DL transformation for $\eta>\eta^{*}$, where $\eta^{*}$ was the critical value of $\eta$ estimated as $\eta^{*} \sim \tau_{\text {closure }} / \tau_{\text {esc }}=1$.

Importantly, in the TC/DL there was no "gradient inversion" in that system - that is, water contents were always lower in the edges than in the nodes, and switching between the patterns depended only on whether the iron cations preferred to move "vertically" or "laterally". In the present work, the ions always move "laterally" ("vertical" transport is negligible) - the directions of their motions are controlled by the relative water outflow from the $\underline{3}$ and $\underline{4}$ nodes. As a result, the overall water gradients along a $\underline{3}-\underline{4}$ edges can be either convex or monotonically decreasing from $\underline{4}$ to $\underline{3}$, and pattern switching occurs when the derivative of the water content along the edge, $d \rho(x) / d x$, changes sign. The dependence on the derivative (and not only one the slowlyvarying $\rho$ function) makes the present system much more sensitive to the properties of the substrate. While the TC/DL transition required large (macroscopic) changes in the system's geometric parameters (e.g., thickness of the gel layer or network's dimensions), 
the symmetric/asymmetric switch describe here is sensitive to the conformational changes of gelatin.

(a)

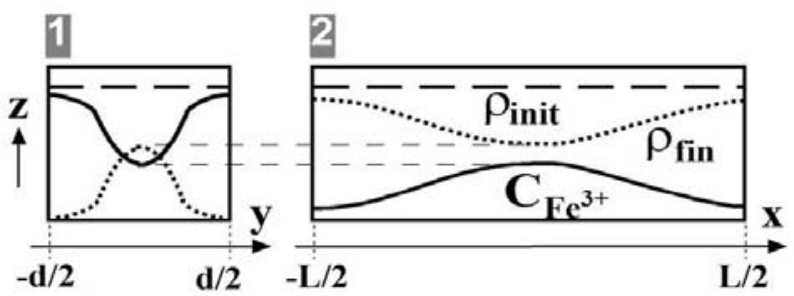

(b)

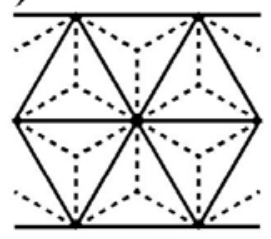

TC

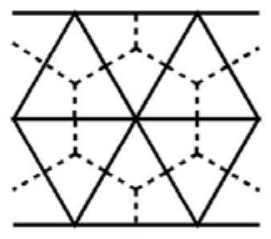

DL
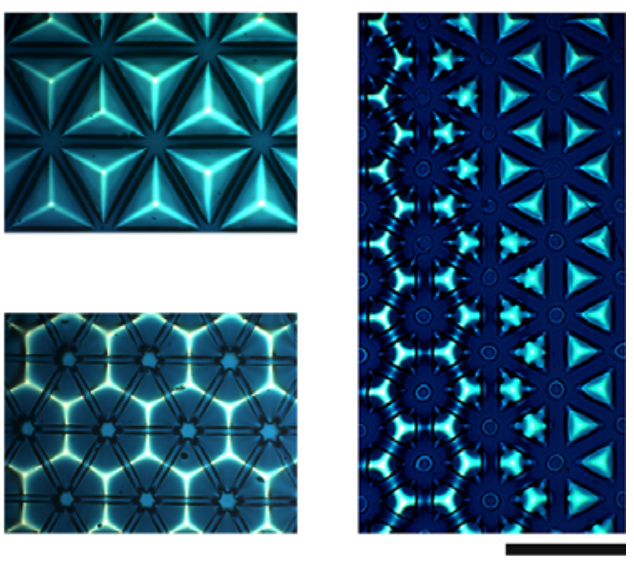

Fig S1: RD patterns in networks with one type of nodes. (a) The schemes show qualitative water content profiles across an edge (1, left) and along it (2, right). The profiles are always symmetric and -- along the edge -- have a minimum at its center. (b) Two examples of color patterns (tile-centered, TC, and a dual-lattice, DL) developed from identical stamped lattices of equilateral triangles on gels varying in thickness by $\sim 1$ $\mu \mathrm{m}$. (scale bar $=500 \mu \mathrm{m}$ ) The rightmost picture illustrates a transition between the two geometric solutions on a gel of thickness continuously varying from $\sim 10 \mu \mathrm{m}$ in the topright corner (TC solution) to $\sim 35 \mu \mathrm{m}$ in the bottom-left corner (DL solution).

\section{S2. Network Design Rules}

(i) Topology: Since the mechanism of the symmetric/asymmetric pattern switching requires that different nodes of the network dehydrate at different rates (thus facilitating the "gradient inversion" phenomenon; cf. S1 above), the stamped micronetwork should be composed of at least two types of nodes characterized by different rates of water outflow. The critical value $\alpha^{*}$ of water absorptivity at which the switch occurs depends the relative degrees of the nodes according to $\left(\sigma_{n}-\sigma_{m}\right) \alpha^{*} \approx$ const (in the text, $n=3$ and $m=4)$ where $\sigma$ increases with decreasing node degree. This means, for example, that if $n$ and the length of the $\underline{n}-\underline{m}$ edge are kept constant, and if $m$ is increased (e.g. to a five foldnode), the network will switch at a lower value of water absorptivity. 


\section{(ii) Dimensions:}

Firstly, the pattern-switch requires that diffusion of ions along the network's edges dominates that in the direction across the edges - in other words, the $h$ and $d$ dimensions (see Fig. S2) must be sufficiently larger than $w$ (otherwise, the ions would migrate into gelatin from the edges to give TC-like solutions irrespective of the properties of the substrate). We have experimentally estimated that the $h / w$ and $d / w$ ratios should be larger than $\sim 4$.

Secondly, the dimensions $d$ and $h$ should be similar, although their absolute values can differ (as predicted by the approximate switching condition given in the text, $\left(\sigma_{3}-\sigma_{4}\right) \alpha^{*} \approx$ const, which does not depend on the absolute network dimensions). On the other hand, when $d$ is significantly longer than $h$, (or vice versa) irregular/mixed patterns appear, since the nodes cannot "pump" all the ions from the long edges. For example, we observed that for fixed values of $w$ and $h$, there existed a limiting value, $d_{\max }$ above which the patterns had a significant proportion of mixed (symmetric/asymmetric) as well as TC and DL solutions (Fig. S3). For $w=50 \mu \mathrm{m}$, and $h=350 \mu \mathrm{m}$ the limiting length was $d_{\max }$ $\approx 500 \mu \mathrm{m}$; for $w=50 \mu \mathrm{m}$, and $h=400 \mu \mathrm{m}$ it was $d_{\max } \approx 570 \mu \mathrm{m}$.

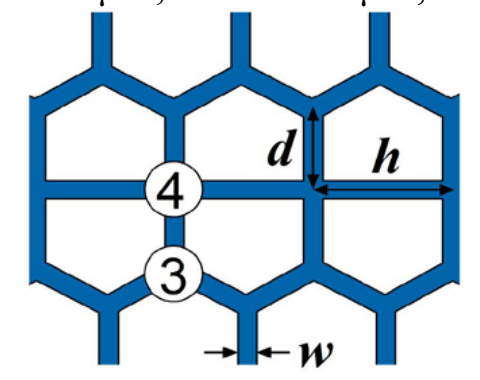

Fig S2: Characteristic

dimensions, $d, h$, and $w$, of

the stamped

micronetwork.

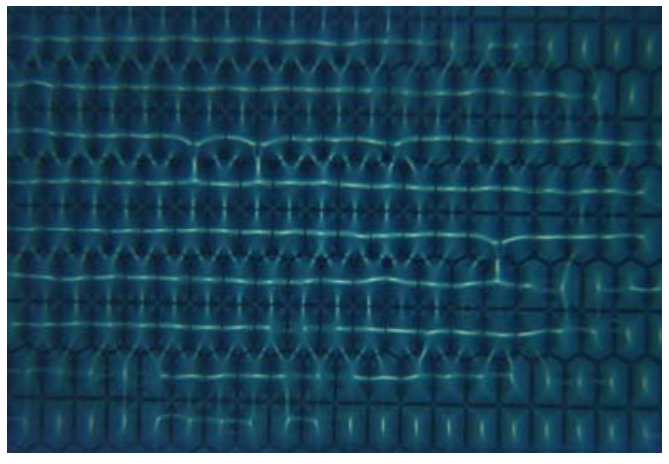

Fig S3: Example of an

irregular/mixed pattern produced

for $w=50 \mu \mathrm{m}, h=400 \mu \mathrm{m}$ and

critical $d_{\max } \approx 570 \mu \mathrm{m}$.

\section{S3. References.}

The full reference (4) reads:

Ansari. A.; Berendzen, J.; Braunstein, D.; Cowen, B.R.; Frauenfelder, H.; Hong, M.K.; Iben, I.E.T.; Johnson, J.B.; Ormos, P.; Sauke, T.B.; Scholl, R.; Schulte, A.; Steinbach, P.J.; Vittitow, J.; Young, R.D.; Biophys. Chem. 1987, 26, 337-355. 